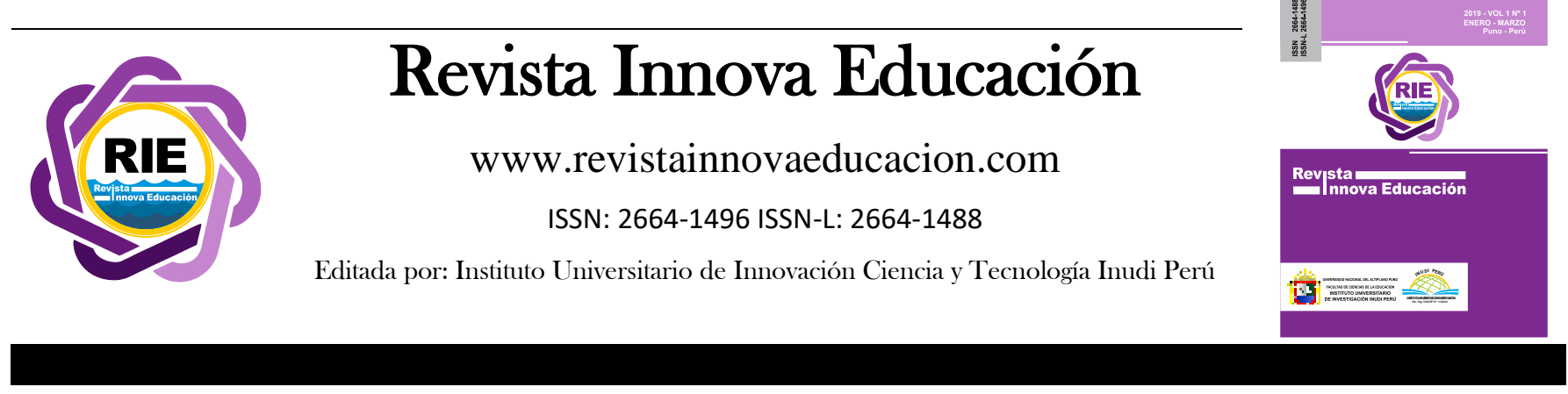

\title{
Revisión del concepto de identidad profesional docente
}

\author{
Review of the concept of teaching professional identity
}

\author{
Sergio Olave ${ }^{1}$ \\ Universidad Americana de Europa, Cancún - estado de Quintana Roo, México \\ https://orcid.org/0000-0001-6900-0277
}

DOI: https://doi.org/10.35622/j.rie.2020.03.001

Recibido 11/03/2020/ Aceptado 25/07/2020 Publicado 25/07/2020

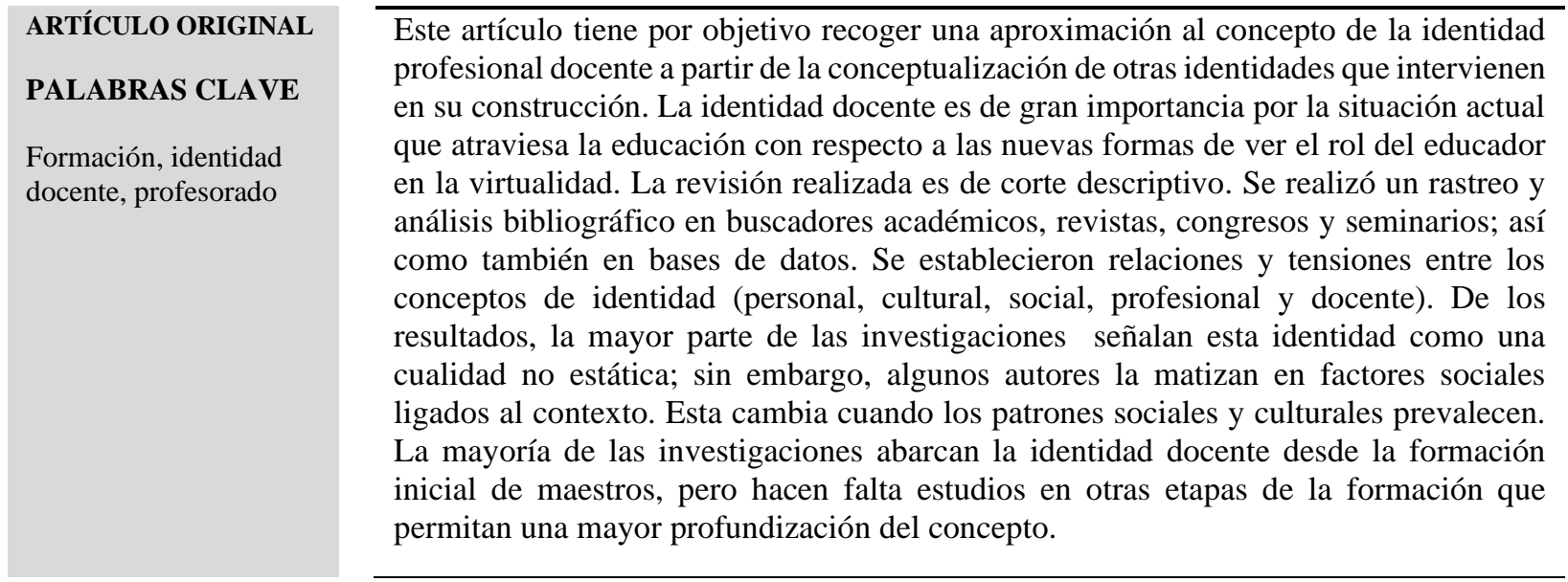

KEYWORDS

Teacher identity, training, teaching staff

\footnotetext{
${ }^{1}$ Correspondencia: sergiolave_90@hotmail.com
}

\begin{abstract}
This article aims to collect an approximation to the concept of the teaching professional identity from the conceptualization of other identities that intervene in its construction. The teaching identity is of great importance due to the current situation that education is going through with regard to new ways of seeing the role of the educator in virtuality. The narrative is carried out for this review. A bibliographic search and analysis was carried out in academic search engines, magazines, conferences and seminars; as well as in databases. Relationships and tensions were established between the concepts of identity (personal, cultural, social, professional and teaching). From the results, most of the studies indicate this identity as a non-static quality; however, some authors qualify it in social factors linked to the context. It changes when social and cultural patterns prevail. Most papers cover teacher identity from the initial teacher training, but studies are needed at other stages of the training that allow a deeper understanding of the concept.
\end{abstract}




\section{INTRODUCCIÓN}

En la actualidad los docentes como profesionales de la educación han tenido que enfrentarse a una serie de patrones culturales, sociales, políticos y económicos que han hecho que su identidad como individuos se vea afectada, adoptando una identidad profesional cliché establecida por la sociedad. Esta revisión del concepto busca señalar el concepto de identidad profesional docente más allá de una serie de comportamientos y características que el maestro debe poseer y seguir cuando decide optar por esta profesión. Por tal motivo, el objetivo de esta revisión del concepto es comprender la identidad profesional del docente como una identidad cambiante que se nutre de otras identidades y que no debería ser tomada de forma estática, a través del tiempo.

La identidad está ligada al contexto y por ende no puede desligarse de su historicidad; sin embargo, esta siempre ha sido blanco de ataques, no bélicos producidos por alguna guerra, sino los ocasionados por las costumbres subyacentes de una sociedad inmóvil que se niega a ver al otro de forma diferente. En ese mismo sentido, es importante que cada individuo conozca su rol en la sociedad, de tal manera que pueda vivir de forma coherente. De allí, que la auto-comprensión es un proyecto del ser humano que nunca termina y es entonces cuando se entiende que la identidad no es estática; una fuente que emerge de lo subjetivo; es entonces, cuando las emociones afectan al ser y hace que éste mute y se transforme (Barreto, 2018; Echarte y Erquiaga, 2019; Lagos y Polster, 2018; Crespo, 2019).

Por otra parte, la memoria se convierte en aquel recurso que conserva nuestra identidad en lo individual y lo colectivo (Crespo, 2016; Fernández, 2017). De esta manera, se obtienen y transforman aquellos recuerdos en el tiempo; por consiguiente, la conciencia se vuelve más aguda cuando nos domina algún tipo de afecto o entramos en conflicto con alguna costumbre; estas situaciones generan un valor en ese algo que nos afecta (Alvarado, 2016; López, 2016). Así mismo, unos recuerdos soportan a otros y en esta singularidad no hay un solo modelo, puesto que aquello que se toma del entorno, se toma para crear una nueva personalidad irrepetible y que solo puede ser entendida por sí misma (Vicente, 2019; de la Calle y Méndez, 2018).

Por lo anterior, así como hay una identidad personal y única, en la construcción de dicha identidad también intervienen factores culturales, sociales y profesionales; siendo estos últimos el fin último de la escuela. El ser humano es biológico, psicológico y a su vez una criatura social y moral (Shoemaker y Tobia, 2019). La identidad cultural toca el pasado y futuro de cada persona y son las tradiciones determinantes en la construcción de identidad de un pueblo; debido a que, estas pueden perdurar o desaparecer en las dinámicas de intersubjetividad de cada individuo (Cruz, 
Echevarría y Gutiérrez, 2019). En otro orden de ideas, la identidad debe ser entendida como un proceso de socialización que permite al sujeto encontrar su propia singularidad. Desde ciertas posturas, se afirma que la cultura es heredada y se transmite de generación en generación, sin embargo, la idea constructivista señala que la identidad cultural es un constructo social. De igual manera, existe una otredad que se potencia cuando hay patrones comunes en una relación con la diferencia.

La identidad cultural da respuesta a intereses, normas y características que comparten ciertos grupos. Muchas de las veces, la tradición se moviliza dentro de una reconstrucción para mantenerse en la modernidad; por lo tanto, el sentimiento de pertenencia a determinado grupo social con ciertos rasgos culturales diferentes a los demás, constituye una identidad cultural sin confundir la anterior acepción con el sentimiento comunitario, que se expresa en manifestaciones culturales (Chenet, Arévalo, y Palma, 2017; Gómez, 2019; Cuesta, Jústiz, y Argilagos, 2019; Cascante, 2004; Ottone, 2017; Ortega, 2018).

Por otro lado, la identidad social se legitima desde las relaciones que se dan en aquellos grupos culturales y entra en tensión cuando hay una relación con otras culturas sin importar territorio o lenguaje. Esta identidad social está sujeta a matices y al igual que la identidad cultural, no es estática. Así, se hablaría de un yo social consciente de su cultura, de sus gustos e intereses, pero vulnerable a la reestructuración y acomodamiento que se da en la relación con los otros (Cantor, 2018; Salas, 2019; Labrada, Barba-Tellez y González, 2016; Salcedo, Obando y Ordóñez, 2018; Acosta y Vargas, 2017). La universidad es un espacio que permite el encuentro de diferentes identidades culturales y el desarrollo de la identidad social, cuya característica en común es la de educarse; no obstante, aquí surge otro término que, a diferencia de la generalidad, subyace en los gustos dentro de ese espectro amplio de la educación y a la cual se denomina: la identidad profesional.

La identidad profesional se desarrolla a partir de la relación de la identidad personal y la historicidad de la profesión, a esto se le denomina, vocación (Callata, Morales y Arias, 2017). De igual modo, intervienen aspectos familiares y escolares, puntos críticos en el proceso de construcción de identidad profesional. Las experiencias que se enmarcan en estas dos dimensiones repercuten de forma directa en los primeros momentos de la formación de la identidad profesional (Pérez y Martínez, 2018). 
Tabla 1 Concepto y características

\begin{tabular}{|c|c|c|}
\hline $\begin{array}{l}\text { Ejes de } \\
\text { análisis }\end{array}$ & Descripción & Características \\
\hline $\begin{array}{l}\text { Identidad } \\
\text { personal }\end{array}$ & $\begin{array}{l}\text { Se puede definir como las } \\
\text { condiciones dadas por la } \\
\text { continuidad psicológica entre las } \\
\text { etapas personales, en lo } \\
\text { relacionado a la persistencia tem- } \\
\text { poral. }\end{array}$ & $\begin{array}{l}\text {-Corporalidad } \\
\text {-Temporalidad } \\
\text {-Continuidad psicológica }\end{array}$ \\
\hline $\begin{array}{l}\text { Identidad } \\
\text { social }\end{array}$ & $\begin{array}{l}\text { Los aspectos de un individuo } \\
\text { que se basan en la pertenencia a } \\
\text { grupos sociales, junto con sus } \\
\text { aspectos psicológicos, } \\
\text { emocionales, entre otros }\end{array}$ & $\begin{array}{l}\text {-Dinámica } \\
\text {-Tiene un origen y desarrollo sujeto a condiciones } \\
\text { históricas concretas } \\
\text {-Se enriquece en la médica que el ser humano se } \\
\text { desarrolla y adquiere nuevos conocimientos que lo } \\
\text { etiquetan en un grupo social determinado. } \\
\text {-Se basa en la vida cotidiana }\end{array}$ \\
\hline $\begin{array}{l}\text { Identidad } \\
\text { cultural }\end{array}$ & $\begin{array}{l}\text { Definida históricamente a través } \\
\text { de múltiples aspectos en los que } \\
\text { se plasma la cultura, la lengua, } \\
\text { las relaciones sociales, ritos y } \\
\text { ceremonias propias, o los } \\
\text { comportamientos colectivos, los } \\
\text { sistemas de valores y creencias. }\end{array}$ & $\begin{array}{l}\text { - Los medios de socialización primarios como la } \\
\text { familia, la escuela y la iglesia intervienen en su } \\
\text { configuración. } \\
\text {-Hay una postura esencialista y otra constructivista. }\end{array}$ \\
\hline $\begin{array}{l}\text { Identidad } \\
\text { profesional }\end{array}$ & $\begin{array}{l}\text { El conjunto de hábitos que una } \\
\text { persona ha desarrollado en el } \\
\text { espacio de su trabajo. Abarca la } \\
\text { experiencia laboral y define en } \\
\text { su gran mayoría la identidad } \\
\text { personal. }\end{array}$ & $\begin{array}{l}\text {-Se va estructurando desde la escuela. } \\
\text {-Se fortalece durante la formación profesional. } \\
\text {-Se consolida con el ejercicio profesional } \\
\text {-Los intereses y las aptitudes vocacionales son su } \\
\text { base. }\end{array}$ \\
\hline $\begin{array}{l}\text { Identidad } \\
\text { docente }\end{array}$ & $\begin{array}{l}\text { Es la identidad de una persona } \\
\text { que trabaja en un área de trabajo } \\
\text { específica de la educación. Se } \\
\text { considera un proceso en continua } \\
\text { transformación compuesto por } \\
\text { diferentes dimensiones. Es única } \\
\text { y no puede sustituirse porque se } \\
\text { crea y se va evolucionando } \\
\text { durante la vida. }\end{array}$ & $\begin{array}{l}\text {-Relación Mundo profesional y Universitario. } \\
\text {-Relación teoría y práctica. } \\
\text { - Vinculo conocimiento y acción }\end{array}$ \\
\hline
\end{tabular}

En ese orden de ideas, podría pensarse entonces que la identidad profesional se conforma a partir de la relación social que se da entre un colectivo determinado, condicionado a características culturales que involucran dimensiones históricas emocionales, afectivas, familiares y escolares. Entonces, la identidad profesional docente, no solo se enmarca en una identidad meramente personal, de vocación o social, o por interacción entre culturas en determinado contexto; sino también, por la memoria y las relaciones intersubjetivas que experimenta el individuo en su entorno y vida cotidiana. 


\section{MÉTODO}

El objetivo de esta revisión fue identificar los aspectos relevantes sobre el concepto de identidad profesional docente y las aproximaciones teóricas sobre este. De esta manera, para la búsqueda de los documentos bibliográficos se realizó una exploración en diferentes repositorios y bases de datos y se acudió a varias fuentes documentales como Scopus, Mendeley y ScienceDirect. Se utilizaron descriptores correspondientes a identidad, identidad personal, identidad cultural, identidad social, identidad profesional, identidad profesional docente, escritura científica, revisión de literatura y lectura crítica, entre el periodo del año 2016 y principios del año 2020. Los descriptores se combinaron de diferentes formas con la finalidad de ampliar los hallazgos. Del mismo modo, se realizó una búsqueda en google académico con dichos términos incluyendo citas y patentes en cualquier idioma.

A partir de lo anterior se partió de la pregunta ¿qué se sabe de la identidad (personal, cultural, social, profesional, profesional docente) y cuáles son sus características? En seguida se determinaron los tipos de documentos (primarios y secundarios). Se seleccionaron 50 artículos que dieran cuenta de dichos conceptos y se clasificaron según los criterios de inclusión y exclusión correspondientes a las categorías de los descriptores. Se agruparon los documentos en tipologías de identidades en el siguiente orden: identidad (I), identidad personal (IP), identidad cultural (IC), identidad profesional (IPR) e identidad profesional docente (ID). A continuación, se hizo el correspondiente examen de cada una de las tipologías, para encontrar elementos en común y tensiones entre los conceptos. Finalmente, se enunciaron algunas hipótesis y conclusiones alrededor del concepto. La literatura estudiada se enmarcó en una búsqueda a nivel global con preferencia en idioma español en correspondencia a lugares donde hay presencia de Escuelas Normales.

La revisión de literatura permite hacer un compendio de estudios con la finalidad de analizar y discutir un problema (Sánchez, 2011), en este caso del concepto de identidad profesional docente. Se realizó una revisión descriptiva, que permita al lector tener una mirada más hacia un concepto (Day, 2005 en Guirao, Olmedo y Ferrer, 2008). En este caso, se hace una aproximación a la identidad del maestro, desde una revisión del concepto de identidad, pasando por la identidad personal, cultural, social y profesional. El análisis de los documentos siguió una lectura crítica para determinar los argumentos y las fortalezas y debilidades de las ideas planteadas (Garcés y Duque, 2007). 


\begin{tabular}{lll}
\hline $\begin{array}{l}\text { Universo de análisis } \\
\text { (población) }\end{array}$ & \multicolumn{1}{c}{$\begin{array}{c}\text { Ejes de } \\
\text { investigación }\end{array}$} & Unidades de investigación \\
\hline $\begin{array}{l}\text { Identidad Profesional } \\
\text { Docente }\end{array}$ & $\begin{array}{l}\text { Identidad personal } \\
\text { Identidad social } \\
\text { Identidad cultural }\end{array}$ & Concepto y características \\
& $\begin{array}{l}\text { Identidad } \\
\text { profesional }\end{array}$ & \\
& Identidad docente & $\begin{array}{l}\text { Qué y cómo se concibe la identidad } \\
\text { docente. } \\
\end{array}$ \\
& La importancia de la identidad docente.
\end{tabular}

Tabla 2 Unidades de investigación

\section{RESULTADOS}

\section{La identidad cambiante del maestro}

Muchos asumen la identidad profesional y la identidad docente como una misma; en cambio, esta última se enmarca en una identidad establecida con la expresión "debe ser", a diferencia de otras profesiones; el maestro "debe ser" o asumir una identidad preestablecida, de lo contrario no puede llamarse maestro. Estos constructos de identidad están arraigados a características culturales y de contexto, por lo tanto, no es construida por quien desempeña la labor de docente, sino que se asume como una identidad ajena que se encasilla en cánones sociales, del ejemplo y de su rol en la sociedad. La cuestión aquí es determinar si el maestro tiene una identidad preestablecida enajenada, contrario a lo que presentaban anteriormente algunos autores sobre el término. Entonces, deberíamos preguntarnos si quien asume como proyecto de vida ser maestro, ¿Debe abandonar su identidad personal? ¿Hay una identidad social y cultural preestablecida para el maestro?

La identidad profesional docente es un proceso de construcción propio, resultado de procesos de socialización que se fortalecen en el ejercicio (Bolivar, Domingo y Pérez, 2014). A través de la imaginación, los individuos forman sentidos, significados y perspectivas que fortifican sus identidades con nuevas dimensiones en el tiempo. (Yazan, y Peercy, 2018). Asumiríamos entonces que el docente necesita de la imaginación para edificar su identidad como profesional. Adicionalmente, se identificó que la identidad es negociada, a través del lenguaje y el discurso (Aristizábal y García, 2017). 
Los discursos sobre la construcción de la identidad profesional docente basan su fundamentación en la percepción de la conciencia personal como compromiso de buena voluntad y la percepción del compromiso moral, este último pensado en lo que se supone que es correcto, que es asumir responsabilidad sobre el alumnado, hacerse cargo del otro en su integralidad, se hablaría de una ética del cuidador. La identidad del maestro pareciera ser una forma de respuesta a las expectativas de los otros grupos sociales. Es evidente entonces, que la identidad de un individuo que trabaja en el campo de la educación, en consecuencia siempre está en continua construcción y se va transformando según las experiencias vividas; ahora bien, cobra vital importancia el reconocer la singularidad de cada individuo en el ejercicio de la docencia y el reconocimiento de la profesión en determinado contexto y grupo social (Pérez y Quijano, 2018; Bolívar, Fernández y Molina, 2004; Alfaya, Moya, Pérez, y García, 2019; Serrano y Pérez, 2018).

Para la consolidación de la identidad profesional docente es necesario reconocer el valor que las emociones y estado de ánimo dan a ésta, puesto que son indispensables para el ejercicio y la práctica del maestro (Buitrago y Cárdenas, 2017). Las emociones y conductas de los maestros se proyectan a los demás sectores sociales; de esta manera, se tipifica, se estereotipa al maestro como alguien que debe ser paciente, respetuoso, ética y moralmente correcto, olvidando su origen y naturaleza humana, más allá de un refinamiento o un ejemplo de difícil imperfección. El maestro, se reconfigura a patrones históricos que subyacen en la cultura, pues el maestro no es digno de dicha identidad, no tiene derecho a la equivocación, a sentir decepción, tristeza, frustración y emociones alternas a las que se deben sembrar en el alumnado. Por tanto, pierde su naturaleza primera, cambia de piel, muta a otro ser paradójicamente libre y construido por otros. El maestro pierde su identidad primera para asumir otra social y culturalmente aceptada.

A diferencia de otras profesiones en la sociedad, el maestro debe asumir una identidad que no siempre le corresponde. Veamos el ejemplo de un médico: puede que recomiende a sus pacientes dejar de fumar, pero ser un fumador activo, o sugerir ejercicio y dieta balanceada a pesar de no cumplir con ninguna de las anteriores; además, el maestro es un ejemplo que no puede salirse de la regla o ser la excepción, puesto que es tomado como el referente del ciudadano ejemplar, que todos deberían ser, pero que en la actualidad nadie quiere ser, debido a que se ha perdido la profesionalización docente y se ha desvalorizado el magisterio a causa de factores, sociales, económicos, políticos y culturales. Las transformaciones en la identidad profesional docente no se dan de una manera aislada y el contexto interviene de forma directa en esta configuración (Aristizábal, 2019). 
Las barreras de género han permeado las profesiones desde mucho tiempo atrás; las diferencias socioeconómicas y culturales que subyacen en la práctica y en los discursos ha estado más abogada a los hombres, dándose una segregación laboral, señalando las ocupaciones y oficios a las que está determinado el género femenino. De esta manera, las mujeres siempre han estado relegadas como cuidadoras desde la maternidad y esto ha hecho pensar que el hombre no está en condiciones de cuidar al infante en ciertas etapas de desarrollo y que de por sí representa cierto peligro desde los constructos sociales que se han dado, a partir de eventos desafortunados de abuso o agresión.

La feminización de la labor docente ha influenciado también la identidad profesional docente para los hombres, quienes ante los prejuicios sociales, tienden a desarrollar una identidad profesional más resistente para lidiar con ciertas concepciones; de igual forma, se puede apreciar una masculinización en las ciencias exactas en la educación. Por lo anterior, se hace necesario pensar también, que la identidad del maestro está condicionada por el género y los roles históricos que han desempeñado ciertas profesiones (Varilla, 2016). Hoy, las barreras de género están quebrándose en las profesiones; aun así, las presunciones de ciertos modelos machistas y feministas interfieren en la construcción de la identidad de quien decide optar por ser docente. Lo anterior se puede apreciar, en que hay muy pocos o casi ningún hombre en educación preescolar y muy pocas mujeres en el campo de la física y las matemáticas.

En la actualidad, se está presentando una crisis de identidad docente (Cervantes y Dengo, 2019), los términos pedagogía y didáctica han entrado a jugar un rol importante para determinar la identidad del maestro; puesto que, aquel profesional de cualquier otro campo diferente a la educación que mantenga un contacto directo con la pedagogía y la didáctica, tiende a asumir su identidad profesional a una identidad profesional docente. Por ende, deberíamos asumir que el profesional con un grado de pedagogía y didáctica se convierte en maestro y asume dicha identidad. De este modo, como se evidencia en la universidad, en el programa de arquitectura no existe un profesor que se haya identificado desde su vocación como maestro de arquitectura, sino como arquitecto, pero por circunstancias de su interacción social tuvo una aproximación a la pedagogía, se convierte en profesor de arquitectura.

No hay individuos que estudien para ser profesores de medicina o derecho, estos últimos asumen esta identidad docente cuando se empiezan a desenvolver como profesores, son médicos enseñando a otros a ser médicos, y abogados enseñando a otros a ser abogados. "las nuevas condiciones y orientaciones de la política educativa en que se demanda un tránsito de un modelo burocrático a una dirección pedagógica, encaminada a la mejora de los aprendizajes y de los 
resultados del establecimiento escolar, de acuerdo con las orientaciones reflejadas en la literatura internacional” (Botía y Real, 2016, p.7), conocer las motivaciones y preocupaciones permite conformar una identidad docente propia.

La identidad del maestro también se ve afectada por la relación que se da entre el centro educativo y las familias (Rogero, 2007 en Santamaría, Torrego y Ortiz de Santos, 2018), la libertad que surja de estas relaciones permitirá equilibrar la identidad personal y la identidad docente en el individuo. Los Movimientos de Renovación Pedagógica han permeado al profesorado como colectivo, señalando que el docente debe tener ciertas actitudes y aptitudes con el fin último de crear personas integrales y críticas. Pero, cabe señalar que dentro de estas actitudes debe estar el reconocimiento como docente y como ser único con identidad particular para poder formar a los estudiantes en ámbitos emocionales bien estructurados; pues un maestro que no tenga identidad docente, no es feliz en su trabajo, y por ello no es consecuente de su labor, así no podrá construir seres más allá de la academia y la epistemología de la disciplina.

Puede afirmarse entonces que el profesorado no posee una identidad estable, que por el contrario es plástica y polimorfa según elementos históricos y de contexto que siempre están en el diario vivir del docente (Jarauta y Pérez, 2017). "La complejidad y relevancia de este objeto de estudio es parte de un proceso que se organiza durante la escolaridad del sujeto y se configura durante la carrera, es decir, se adquieren en instancias formativas en los procesos mismos de enseñanza aprendizaje, están implícitos en la currícula, en las prácticas y en los modos de enseñar y aprender” (Sánchez y González de Álvarez, 2019, p.3).

La tensión entre las políticas gubernamentales y la profesión docente es otra dimensión que merece ser analizada. Si bien, el maestro es consciente en su formación de los desafíos que debe enfrentar, es en la práctica cuando reconoce la realidad que se moviliza en los discursos de los formadores de profesorado. La creencia de un estatus profesional frente a las otras profesiones se ve opacado por las brechas sociales y económicas del sector educativo. El pensamiento utópico de querer cambiar una sociedad para destruir desigualdades se ve abocado a una crisis profesional de auto-reconocimiento como eje transformador y emancipador de la sociedad. Lo anterior puede fortalecer o reconfigurar una identidad que se creía sólida.

El encuentro con una realidad de forma directa conlleva a que muchos docentes pierdan su horizonte primero y se refugien en una identidad pesimista donde describen su labor como algo mecánico y regresan nuevamente a su identidad personal y social. Esto se ve claramente en el sector público donde las inequidades, los ambientes y las apuestas pedagógicas están dados para regular índices de cobertura y no de educación de calidad. Otros docentes, en cambio, fortalecen 
su identidad profesional abandonando su identidad como persona, sus motivaciones primeras, sus gustos, sus sueños personales, pasando de su prioridad primera del yo a la otredad como fuente de idealización y nuevas motivaciones.

En consecuencia, se puede ver cada vez menos jóvenes interesados en seguir una carrera de profesorado (Eguren y Belaunde, 2019). Las crisis de identidad en los jóvenes son cada vez más frecuentes. La dinámica escolar hace que los estudiantes no se vean identificados con sus maestros, generando una brecha gigantesca en lo que quiere el maestro y lo que quiere el estudiante.

Se hace entonces urgente derrumbar la imagen del maestro como un ser inerte carente de emociones y lleno de virtudes y perfección, por el contrario construir una autoimagen común que no cree una forma de ser docentes sino múltiples formas de serlo y de vivir la profesión (González y Sánchez, 2017), rescatando las singularidades, para que cuando el maestro hable de sí mismo pueda hacerlo refiriéndose a su propia identidad, a lo que es, a lo que cree que es y no a lo que se supone debe ser.

Por último, el docente responde a un contexto de forma personal, De esta forma, hablaríamos de subidentidades en las relaciones que se establecen en dicho contexto, que si bien pueden darse de forma armoniosa o de conflicto también son dimensiones que reconfiguran la identidad del maestro (González, 2018; Juárez y Hernández, 2017; Alfonzo y Ortiz, 2019). En algunos estudios encontrados en esta revisión, los profesores en formación inicial relacionan su identidad con el conocimiento de la disciplina y manifiestan que las emociones inciden en los escenarios de la escuela, pero que el docente debe ser ajeno a ellas. Entre tanto, se espera a un docente sabio y creativo que pueda reflexionar para modificar su realidad inmediata (Cruz y García, 2018; Laspina, 2018).

El maestro en formación integra hábitos previos que definirán su identidad profesional, y así afiance su concepto del ser profesor; esto configurará docentes que abandonen su profesión, otros que optarán por el profesorado por admiración a la profesión, aquellos que se resignan a la profesión escogida y finalmente aquellos que adapten sus motivaciones y expectativas a lo que encuentren en el medio (Reyes y García, 2019; Mayo, García y Rodríguez, 2017; Quilaqueo, Quintriqueo, Segundo y Riquelme, 2016). En resumen hay una diversidad de condiciones sociales y psicológicas que se relacionan entre sí, de esta manera hay procesos de orden biológico, psicológico y social (Rodríguez y Pedrajas, 2016), pero también, dimensiones personales, 
históricas y culturales en la construcción de una identidad docente, a diferencia de las otras identidades sociales.

\section{CONCLUSIONES}

Se encontraron diferencias entre conceptos al igual que similitudes que coadyuvaban a definir que la identidad profesional del maestro no es estática sino cambiante y que esto depende de factores biológicos, psicológicos, sociales, culturales, personales y profesionales. No se puede hablar de una identidad profesional del maestro, puesto que, ésta es reflejo de la interacción en el contexto y su sentido frente a la profesión. Esta identidad debe ser entendida entonces bajo diferentes percepciones que no son iguales en el tiempo, tampoco es una identidad innata que nace con la persona, aquello que llaman vocación no es más que el conjunto de patrones que se solidifican en la interacción con la realidad y los diferentes grupos sociales, donde elementos comunes convergen para soportar una identidad docente que se está construyendo.

Ninguna persona puede construir una identidad basada en lo que otros dicen. La identidad del maestro es una identidad para sí, complementaria a los procesos sociales y las relaciones que emergen; la significación que el maestro otorga a su identidad se configura en una intersubjetividad de idas y venidas en el marco de la subjetividad y la objetividad de su labor.

Para comprender la identidad profesional docente es necesario comprender los conceptos de identidad personal, social y cultural que conforman la identidad del maestro. Es por medio de las relaciones sociales que se reconocen características que se han atribuido a cada grupo, a través de la historia; así, la identidad profesional hace parte de las identidades sociales donde se valoran o desestiman según el contexto y la cultura. Pensar en la profesión docente más allá de una labor con sentido social obliga a cuestionarnos qué la hace tan ajena a las demás y tan difícil de asumir como una sola identidad, pues es una identidad cambiante que no puede ser abordada desde una sola perspectiva, sino reconocida en su complejidad.

Finalmente, la identidad profesional docente no está determinada cuando se elige ser parte del profesorado; sino que se constituye desde el hogar y la escuela. Los maestros no se identifican como maestros en alguna etapa específica de su vida; están en constante aceptación de su rol, en el entramado de relaciones políticas y sociales en la práctica. Una identidad que fácilmente es influenciable y volátil desde las representaciones sociales que se tienen del maestro, a través del tiempo y la historia.

\section{REFERENCIAS BIBLIOGRÁFICAS}


Acosta, H. B., \& Vargas, R. H. T. (2017). Precisiones conceptuales para explicar la historia inicial de la identidad social de algunas profesiones en Colombia. Diálogos de saberes: investigaciones y ciencias sociales, (46), 41-58.

Alfaya, E. G., Moya, M. M., Pérez, A. C., \& García, M. D. L. Á. O. (2019). Construcción de la identidad profesional docente en educación infantil en Córdoba (España). Revista de ciencias sociales, 25(3), 30-41.

Alfonzo, I., \& Ortiz, C. (2019). La identidad profesional docente de profesores de educación superior en La región meseta comiteca tojolabal del estado de Chiapas. Revista Electrónica de Investigación $\quad$ Innovación Educativa-REIIE ISSN: $\quad 2448-556 X, \quad 4(3), \quad 115-130 . \quad$ Consultado de http://cresur.edu.mx/OJS/index.php/CRESUR_REIIE/article/view/441

Alvarado, J. (2016). Identidad personal y ontología de la persona. Universitas Philosophica, 33(66), 77-112. https://doi.org/10.11144/Javeriana.uph33-66.ipop

Aristizábal, A. (2019). Fortalecimiento de la identidad profesional docente en el ámbito personal del profesor. Tecné Episteme y Didaxis: revista de la Facultad de Ciencia y Tecnología, (46), 189-204.

Aristizábal, A., \& García, A. (2017). Fortalecimiento de la identidad profesional docente a través del trabajo en comunidades de desarrollo profesional que trabajan con la historia de las ciencias. Enseñanza de las ciencias, (Extra), 3599-3604.

Barreto, $\quad$ M. (2018). $\quad$ La $\quad$ Identidad. RedPensar, 6(1), 1-11. https://doi.org/10.31906/redpensar.v6i1.128

Bolivar, A., Domingo, J., \& Pérez, P. (2014) Crisis and Reconstruction of Teachers' Professional Identity: The Case of Secondary School Teachers in Spain". The Open Sports Sciences $\begin{array}{llllll}\text { Journal, } & \text { vol. } \quad 7, \quad \text { (Suppl-2, } \quad \text { M4), } \quad 2014, \quad \text { pp. } & \text { 106-112 }\end{array}$ http://benthamopen.com/contents/pdf/TOSSJ/TOSSJ-7-106.pdf

Bolívar, A., Fernández, M. y Molina, E. (2004). Investigar la identidad profesional del profesorado: una triangulación secuencial. [Documento en línea]. FQS Recuperado el 12 de agosto 2006 en http://www.qualitative-research.net/fqs.

Botía, A. B., \& Real, M. R. (2016). Identidad profesional de los directores escolares en España. Un enfoque biográfico narrativo. Opción: Revista de Ciencias Humanas y Sociales, (79), 163-183.

Buitrago, R., \& Cárdenas, R. (2017). Emociones e identidad profesional docente: relaciones e incidencia. Praxis \& Saber, $8(17)$, 225247. https://dx.doi.org/10.19053/22160159.v8.n17.2018.7208 
Callata, M., Morales, A., \& Arias, W. (2017). Identidad profesional y preferencias profesionales en estudiantes de la escuela profesional de administración de negocios de una universidad privada de Arequipa. Revista de Investigación en Psicología,20(1), 147 - 176. https://doi.org/10.15381/rinvp.v20i1.13529

Cantor, M. I., Pérez, E., \& Carrillo Sierra, S. M. (2018). Redes sociales e identidad social. Aibi Revista de investigación, administración e ingeniería, 6(1), 70-77. https://doi.org/10.15649/2346030X.477

Cascante, F. R. (2004). Modernidad e identidad cultural en América Latina. Revista Artes y Letras. Universidad de Costa Rica. 17(2), 237- 255.

Cervantes, G., \& Dengo, M. (2019). Identidad Profesional y formación docente universitaria: Un proceso en construcción desde la mirada del estudiantado. Actualidades Investigativas en Educación, 19 (1), 196-215.

Crespo, M. (2016, July). Fenomenología, sentimientos e identidad. La contribución de la fenomenología husserliana de los sentimientos a la cuestión de la identidad personal/Phenomenology, Feelings and Identity: The Contribution of Husserlian Phenomenology of Feelings to the Problem of Personal Identity. In Anales del Seminario de Historia de la Filosofía, Universidad Complutense de Madrid 33 (2), 605.

Crespo, M. (2019). Emociones morales, revelación de la identidad personal y evidencia del corazón. Quién: revista de filosofía personalista, (10), 137-152.

Cruz, D. S., Echevarría, A. M., \& Gutiérrez, R. P. (2019). Fortalecimiento de la identidad cultural a través del rescate de tradiciones en la niñez/Strengthening cultural identity through the rescue of traditions in childhood. Islas revista de Humanidades y Ciencias Sociales de la UCLV, (192), 177-190.

Cruz, J.P., \& García, A. (2018). 3B006 Las emociones y su relación con la identidad profesional de docentes en formación inicial de química de la Universidad Distrital Francisco José de Caldas. Tecné Episteme y Didaxis: TED, (Extraordin), 1-8. Recuperado a partir de https://revistas.pedagogica.edu.co/index.php/TED/article/view/9247

Cuesta, L., Jústiz, M., \& Argilagos, Z. (2019). La identidad cultural en los escolares de la Enseñanza Primaria. Una alternativa desde las actividades complementarias y extraescolares. Opuntia Brava,11(Especial 2), 134-151. Recuperado a partir de http://opuntiabrava.ult.edu.cu/index.php/opuntiabrava/article/view/907

De la Calle, M., \& Méndez, M. (2018). La influencia familiar en la construcción de la identidad personal. Fundamentos y métodos para la formación permanente de los maestros de 
Educación Infantil. Análisis y prospectiva en la CAM (Doctoral dissertation, Universidad Complutense de Madrid).

Echarte, L., \& de Erquiaga, J. (2019). Del yo narrativo a la identidad personal. Humanidades: Revista De La Universidad De Montevideo, (5), 111-148. https://doi.org/10.25185/5.5

Eguren, M., \& Belaunde, C. (2019). No era vocación, era necesidad: motivaciones para ser docente en el Perú. Instituto de Estudios Peruanos, Documento de Trabajo; 256, 7-63.

Fernández, D. (2017) La identidad personal como imperativo social: Una aproximación desde Foucault y Derrida. Granada: Universidad de Granada. [http://hdl.handle.net/10481/48949]

Garcés, J., \& Duque, E. (2007). Metodología para el análisis y la revisión crítica de artículos de investigación. Innovar, 17(29), 184-194. Retrieved March 01, 2020, from http://www.scielo.org.co/scielo.php?script=sci_arttext\&pid=S0121 $\underline{50512007000100011 \& \operatorname{lng}=\mathrm{en} \& \operatorname{tlng}=\mathrm{es} .}$

Gómez, A. (2019). Identidad cultural y globalización. Revista De Filosofía Odos / ISSN(E): 23228369, 8(10), 67-75. Consultado de http://www.revistaodos.com/revista/index.php/odos/article/view/81

González, R. I. (2018). La construcción y desarrollo de la identidad profesional docente en Educación Infantil: características y peculiaridades en el caso de los hombres.

González, M.; Sánchez, A. (2017). La construcción de la identidad profesional del profesor de educación física durante la formación inicial: Una mirada desde los estudiantes en la carrera del profesorado de Educación Física en el año 2017 de la Universidad Nacional de Tucumán. 12 $2^{\circ}$ Congreso Argentino de Educación Física y Ciencias, 13 al 17 de noviembre 2017, Ensenada, Argentina. Educación Física: construyendo nuevos espacios. EN: Actas (2017). Ensenada: Universidad Nacional de La Plata. Facultad de Humanidades y Ciencias de la Educación. Departamento de Educación Física. Disponible en: http://www.memoria.fahce.unlp.edu.ar/trab_eventos/ev.10102/ev.10102.pdf

Guirao-Goris, J. A., Olmedo Salas, A., \& Ferrer Ferrandis, E. (2008). El artículo de revisión. Revista iberoamericana de enfermería comunitaria, 1(1), 1-25.

Jarauta, B., \& Pérez, M. J. (2017). La construcción de la identidad profesional del maestro de primaria durante su formación inicial. El caso de la Universidad de Barcelona. Profesorado. Revista de Curriculum y Formación del Profesorado, 21(1), 103122.

Juárez, Á. I. R., \& Hernández, F. H. (2017). La reconfiguración de la identidad profesional del docente de educación básica ante sus procesos de evaluación. 
Labrada, A. R. G., Barba Tellez, M. N., \& González, E. (2016). Identidad Social y Cotidianidad, Categorías Esenciales en la Relación Universidad-Comunidad. Experiencias y Realidades. Educación, Arte, Comunicación: Revista Académica Investigativa y Cultural, (5), 49-58.

Lagos, G., \& Polster, J. (2018). David Hume y el problema de la identidad personal (Doctoral dissertation, Universidad Católica de la Santísima Concepción).

Laspina, A. J. (2018). La Identidad profesional docente: un estudio desde las representaciones de los estudiantes del profesorado en educación primaria.

López, J. B. (2016). Invención y disolución moderna de la identidad personal. Boletín Millares Carlo, (23), 151-165.

Mayo, I. C., García, S., \& Rodríguez, R. C. (2017). Construcción de la identidad profesional en los futuros maestros de infantil. Revista Infancia, Educación y Aprendizaje, 3(2), 612-617.

Ortega, J. C. (2018). Una aproximación al concepto de identidad cultural a partir de experiencias: el patrimonio y la educación. Tabanque. Revista Pedagógica, (31), 244-262.

Ottone, E. (2017). “El concepto de acumulación civilizatoria. Entre universalismo e identidad cultural”. Estudios Públicos (147), 251-262

Pérez, M., \& Quijano, R. (2018). Análisis del discurso de los estudiantes de magisterio sobre la contribución del practicum al desarrollo de su identidad profesional docente. Educatio Siglo XXI, 36(2), 331-352. https://doi.org/10.6018/j/333091

Pérez, J. M. R., \& Martínez, J. D. J. C. (2018). Identidad profesional en estudiantes de comunicación. Revista Iberoamericana de Producción Académica y Gestión Educativa, 4(8), 1-15.

Quilaqueo, D., Quintriqueo, S., \& Riquelme, E. (2016). Identidad Profesional Docente: Práctica Pedagógica en Contexto Mapuche. Estudios pedagógicos (Valdivia), 42(2), 269284. https://dx.doi.org/10.4067/S0718-07052016000200015

Reyes, A. L. P., \& García, P. C. (2019). Los Inicios de la Identidad Profesional en la Formación del Profesorado de Secundaria en Guanajuato, México. Profesorado, Revista de Currículum y Formación del Profesorado, 23(3), 53-70.

Rodríguez, R. S., \& Pedrajas, A. P. (2016). Concepciones sobre la identidad profesional docente en la formación inicial del profesorado de ciencias experimentales de educación secundaria. Educadi, 1(1), 55-71.

Salas, L. (2019). Identidad Social - Cultural en la formación de futuros docentes en la Universidad del Atlántico. Assensus,4(7), 66-81. Recuperado a partir de https://revistas.unicordoba.edu.co/index.php/assensus/article/view/1870 
Salcedo, M. A., Obando, L., \& Ordóñez, E. J. (2018). La identidad social en la ciudad global. Departamento de Ciencias Sociales.

Sánchez, A., \& González de Álvarez, M. L. (2019). La construcción de la identidad profesional del profesor de Educación Física durante la formación inicial. En XIII Congreso Argentino y VIII Latinoamericano de Educación Física y Ciencias (Ensenada, 30 de septiembre al 4 de octubre de 2019).

Sánchez, A. (2011). Manual de redacción académica e investigativa: cómo escribir, evaluar y publicar artículos. Fundación Universitaria Católica de Norte.

Santamaría, N., Torrego, L., \& Ortiz de Santos, R. (2018). Identidad profesional docente y movimientos de renovación pedagógica. Tendencias Pedagógicas, (32), 177-192.

Serrano, R., \& Pérez Gracia, E. (2018). La motivación, la formación psicopedagógica y el practicum facilitadores del desarrollo de la identidad profesional docente. Revista Científica Ecociencia, 5(6), 1-21. Recuperado a partir de http://revistas.ecotec.edu.ec/index.php/ecociencia/article/view/128

Shoemaker, D., \& Tobia, P., Personal Identity (October 1, 2019). Oxford Handbook of Moral Psychology, Forthcoming. Available at SSRN: https://ssrn.com/abstract=3198090.

Varilla, L. (2016). Romper estereotipos de género en la identidad profesional docente: una propuesta de paz. Educación y ciudad, (31), 71-81.

Yazan, B., \& Peercy, M. (2018). "Pedagogically speaking, I'm doing the right things": Three preservice ESOL teachers' identity formation. Teacher Learning and Professional Development, 3(1), 1-18.

\section{Agradecimientos / Acknowledgments:}

Expreso mi agradecimiento a los docentes del Doctorado en Educación de la Unidad de posgrado de la Universidad Americana de Europa, México.

\section{Conflicto de intereses / Competing interests:}

El autor declara que no incurre en conflictos de intereses.

\section{Rol de los autores / Authors Roles:}

No aplica.

Fuentes de financiamiento / Funding:

El autor declara que no recibió un fondo específico para esta investigación.

Aspectos éticos / legales; Ethics / legals:

El autor declara no haber incurrido en aspectos antiéticos, ni haber omitido aspectos legales en la realización de la investigación. 